\title{
Neues Zentrum für Demokratie- und Friedensforschung an der Universität Osnabrück
}

\section{Einleitung}

Am 24. April diesen Jahres wurde an der Universität Osnabrück das im Herbst 2011 gegründete interdisziplinäre Zentrum für Demokratie- und Friedensforschung $(\mathrm{ZeDF})^{1}$ in Anwesenheit zahlreicher Gäste und Studierender von der niedersächsischen Wissenschaftsministerin Prof. Dr. Johanna Wanka offiziell eröffnet.

Bereits in der Vergangenheit war die wissenschaftliche Auseinandersetzung und zivilgesellschaftliche Beschäftigung mit dem Thema »Frieden « ein besonderes Markenzeichen der »Friedensstadt Osnabrück«, die seit April 2002 auch Sitz der Deutschen Stiftung Friedensforschung (DSF) ist. So bestand von 1993-2003 der von der Volkswagenstiftung geförderte »Projektverbund Friedens- und Konfliktforschung in Niedersachsen «, dessen wissenschaftliche Koordinationsstelle unter dem Dach der Universität Osnabrück angesiedelt war (Leitung: Prof. Dr. Günter Bierbrauer). ${ }^{2}$ Darüber hinaus existieren seit 1986 die gemeinsam von der Stadt und der Universität Osnabrück veranstalteten »Osnabrücker Friedensgespräche«, die mehrmals im Jahr prominent besetzte Podiumsdiskussionen zu Fragen der Friedensförderung und Friedenserhaltung organisieren. ${ }^{3}$ Auch am Institut für Migrationsforschung und Interkulturelle Studien (IMIS), am Interdisziplinären Institut zur Kulturgeschichte der Frühen Neuzeit (IKFN) oder am neu gegründeten Zentrum für Interkulturelle Islamstudien (ZIIS) werden zum Teil Themen bearbeitet, die einen Bezug zur Friedens- und Konfliktforschung aufweisen. Zu nennen ist ferner das seit 2009 bestehende Programm einer »Gastprofessur für Frieden und Globale Gerechtigkeit « (Programmverantwortlicher: Prof. Dr. Arnulf von Scheliha). Die GastprofessorInnen sind in Lehre und Forschung an der Universität Osnabrück tätig, nehmen an den Osnabrücker Friedensgesprächen teil und treten in der Öffentlichkeit in

1 Im Internet ist das ZeDF unter www.zedf.uni-osnabrueck.de zu finden.

2 Im Rahmen dieses Projektverbundes wurden Forschungsvorhaben an niedersächsischen Hochschulen in einem Gesamtvolumen von rund 6,9 Millionen Euro unterstützt, die insbesondere die innerstaatlichen und sozialen Ursachen gewaltförmiger Konfliktaustragung sowie die Bedingungen und Möglichkeiten der friedlichen Konfliktbewältigung untersuchten.

3 Siehe auch www.ofg.uni-osnabrueck.de. 
Stadt und Region auf. Sie richten jeweils ein internationales Symposium zu ihrem Thema aus, führen in Kolloquien die schon bestehenden Aktivitäten auf dem Gebiet der Friedens- und Konfliktforschung zusammen und regen neue interdisziplinäre Forschungsinitiativen an.

Dieses Programm ist nunmehr verbunden mit dem neu gegründeten ZeDF, das die übrigen bestehenden Einrichtungen der Universität ergänzen soll. Das ZeDF ist eine Forschungsstelle des Fachbereichs Sozialwissenschaften und versteht sich als institutionelle Plattform für bestehende und künftige Aktivitäten verschiedener Disziplinen im Bereich der Demokratie-, Friedens- und Konfliktforschung einschließlich Lehre und Öffentlichkeitsarbeit. Mit dem ZeDF besteht nun erstmals wieder im Land Niedersachsen eine derartige Forschungseinrichtung.

\section{Inhaltliches Konzept}

Auch wenn sich ein konzises Forschungsprogramm derzeit noch im Diskussionsprozess befindet, ist der Name des Zentrums durchaus von programmatischer Bedeutung. Denn inhaltlich beschäftigt sich das ZeDF sowohl mit den konstruktiven als auch den destruktiven Dimensionen des Zusammenhangs von Demokratie und Frieden. Dabei geht es weniger um die Frage des in den vergangenen Jahren viel diskutierten Theorems vom »Demokratischen Frieden«, das ideengeschichtlich bekanntlich an Immanuel Kants Entwurf »Zum Ewigen Frieden« (1795) anknüpft und in seiner dyadischen Version besagt, dass Demokratien gegeneinander keine Kriege führen. Insbesondere in der Teildisziplin der Internationalen Beziehungen ist dieser Zusammenhang mit Blick auf zwischenstaatliche Kriege immer wieder untersucht und gerade im Kontext gegenwärtiger Demokratisierungspolitiken kritisch beleuchtet worden.

Die Perspektive des Zentrums setzt vielmehr grundsätzlicher an: Es geht um die Disziplinen übergreifende und verschränkende Erforschung der normativen, soziokulturellen und politisch-institutionellen Bedingungen von Demokratie und Frieden - sowohl im Inneren von Gesellschaften (innergesellschaftlicher Frieden) als auch mit Blick auf das friedliche Zusammenleben von Staaten und Gesellschaften (internationaler Frieden). Konzeptionell wird dabei davon ausgegangen, dass Demokratie bzw. Prozesse der Demokratisierung einen möglichen Weg zum Frieden weisen können, der aber keinesfalls bruchlos oder gar zwangsläufig verlaufen muss. Jedenfalls ist die Verwirklichung beider Ziele nicht immer gleichermaßen gewährleistet; Frieden und Demokratie können durchaus in einem Spannungsverhältnis zueinander stehen: 
Demokratie, wie immer auch institutionell ausgestaltet, bedeutet stets einen dynamischen Prozess, der sozialen Wandel und auch die Thematisierung von gesellschaftlichen Konflikten beinhaltet. Demokratie - insbesondere der Prozess der Demokratisierung in Transformations- und Post-Konfliktgesellschaften - zielt mithin auf ein öffentliches Austragen gesellschaftlicher und politischer Konflikte, das unter zugespitzten Bedingungen und Gewalterfahrungen - zu Unfrieden führen kann.

Frieden impliziert hingegen sowohl einen Zustand (im Sinne der Abwesenheit gewaltsamer Konflikte, »negativer Frieden «) als auch ein gehaltvolles, normatives Ziel, bei dem einerseits verschiedene Wertvorstellungen übereingestimmt werden müssen (z.B. Vorstellungen von Gerechtigkeit) und bei dem es andererseits um die »Friedensfähigkeit« von Gesellschaften und somit um die Etablierung von Formen friedlicher Konfliktbearbeitung und -regelung geht (»positiver Frieden«). Demokratie allein führt nicht zum Frieden, schon gar nicht, wenn es sich de facto um formal- oder minimal-demokratische Strukturen handelt, die ggf. sogar als Instrument von Unterdrückung und Marginalisierung von strukturellen Minderheiten (Alexis de Tocquevilles »Tyrannei der Mehrheit«) verstanden werden. Umgekehrt setzt der innergesellschaftliche wie auch der internationale Frieden nicht notwendigerweise Demokratie voraus.

Das Zentrum setzt vor allem an diesem Punkt an und wird in seinen Aktivitäten beide Perspektiven in den Blick nehmen: Zum einen die ambivalente Beziehung zwischen dem Begriffspaar »Demokratie« und »Frieden« in Theorie und Praxis, zum anderen die Frage nach dem Verhältnis von »innergesellschaftlichem« und »internationalem « Frieden und damit verbunden nach friedensgeneigten Ordnungsstrukturen sowohl auf gesellschaftlicher als auch auf weltgesellschaftlicher Ebene. Insofern richtet sich der Blick nicht nur auf europäische, sondern gerade auch auf außereuropäische Gesellschaften, in denen sich teilweise, angesichts von Bürgerkriegen und fragiler Staatlichkeit, die Frage nach den Regeln der Koexistenz verschiedener gesellschaftlicher Gruppen in ganz besonderer Weise stellt. 


\section{Profil}

Am Zentrum für Demokratie- und Friedensforschung sind zurzeit folgende Fachgebiete beteiligt:

- Politische Theorie (Prof. Dr. Matthias Bohlender);

- Staat und Innenpolitik (Prof. Dr. Roland Czada);

- Vergleichende Politikwissenschaft (Prof. Dr. Ralf Kleinfeld);

- Internationale Beziehungen \& Friedens- und Konfliktforschung (Prof. Dr. Ulrich Schneckener, Direktor des ZeDF);

- Evangelische Theologie (Prof. Dr. Arnulf von Scheliha);

- Katholische Theologie (Prof. Dr. Andras Lienkamp).

Die am Zentrum beteiligten Fachgebiete schließen inhaltlich an den konzeptionellen Rahmen an. Demokratie und Frieden sind sowohl Schlüsselbegriffe der Politischen Theorie und Ideengeschichte als auch der Internationalen Beziehungen. In beiden Fachgebieten finden sich unterschiedliche Theorien und Konzepte, die die ideellen und (möglichen) kausalen Verbindungen zwischen beiden Begriffen herstellen, aber eben auch das Spannungsverhältnis zwischen Demokratie/Republik und Frieden kritisch reflektieren. In der Vergleichenden Politikwissenschaft sowie im Fachgebiet Staat und Innenpolitik geht es zudem um die analytisch-empirische Erforschung der Möglichkeiten und Grenzen von Demokratisierungsprozessen bzw. so genannter Demokratieförderung in unterschiedlichen regionalen und gesellschaftlichen Kontexten. Ein besonderes Augenmerk erfährt dabei das Verhältnis von Zivilgesellschaft und Staat ebenso wie die Frage, inwiefern zivilgesellschaftliche Strukturen und zivile Ansätze zur Konfliktbearbeitung genutzt werden können. Die theologische Perspektive schließlich analysiert die sehr unterschiedlichen Voraussetzungen für Genese, Begründung und Geltung der normativen Leitvorstellungen von Demokratie und Frieden kritisch und setzt diese - im Blick auf mögliche Differenzen zwischen den Konfessionen und Religionen - zueinander in Beziehung. Die Religionen thematisieren neben den notwendigen institutionellen und systemischen Bedingungen für innergesellschaftlichen und internationalen Frieden zugleich denjenigen Beitrag, den die einzelnen Akteure dazu leisten können und (in normativer Hinsicht) auch leisten sollen.

Darüber hinaus fügt sich das ZeDF in weitere an der Universität Osnabrück bereits bestehende Schwerpunkte in der Migrationsforschung (Institut für Migrationsforschung und Interkulturelle Studien/IMIS), der Islamforschung (Zentrum für interkulturelle Islamstudien/ZIIS) und der Europaforschung (Jean Monnet Centre of Excellence in European Studies) ein. 


\section{Aktivitäten}

Die erste Schwerpunktaktivität des ZeDF galt im Sommersemester 2012 der Durchführung des seit 2009 bestehenden Programms der »Gastprofessur für Frieden und Globale Gerechtigkeit«, dessen wissenschaftlicher Koordinator Prof. Dr. Arnulf von Scheliha ist. Unterstützt wird das Programm durch das Land Niedersachsen, die Stiftung Westfalen-Initiative, die Deutsche Bundesstiftung Umwelt (DBU) und die DSF. Erste Friedensgastprofessorin im Sommersemester 2010 war Prof. Dr. Dr. h.c. Eveline Goodman-Thau (Jerusalem/Israel). Ihr Wirken war dem Thema »Friede zwischen den Religionen « gewidmet. Friedensgastprofessor im Sommersemester 2011 war Prof. Dr. Neville Alexander (Kapstadt/Republik Südafrika). Er widmete sich den Themen »Friede und Sprachen« und »Ethik und Politik in Transformationsgesellschaften «. Der Mitkämpfer Nelson Mandelas und 1961 an der Universität Tübingen mit einer Arbeit über Gerhart Hauptmann promovierte Linguist verstarb am 27. August 2012. Die Friedensgastprofessur im Jahr 2012 bearbeitete das Thema »Kooperation oder Konflikte um Ressourcen?«. Gleichzeitig mit der Eröffnung des ZeDF startete die Ringvorlesung zu diesem Thema, in deren Verlauf im Sommersemester zahlreiche in- und ausländische Wissenschaftler aus unterschiedlichen Fachgebieten zu Vorträgen in Osnabrück zu Gast waren (u.a. Prof. Dr. Michael Brzoska, Prof. Dr. Dirk Messner, Prof. Dr. Nils Petter Gleditsch, Prof. Dr. Aaron Wolf und Prof. Dr. Olivier de Schutter, UN-Sonderbeauftragter für das Recht auf Ernährung). Die im Rahmen dieser Ringvorlesung gehaltenen Vorträge sollen in Form eines Sammelbandes publiziert werden. Der residente Teil der Friedensgastprofessur wurde durch Dr. Christiane Fröhlich vertreten, die als Fellow am ZeDF arbeitet und u.a. ein Seminar zum Thema »Ressourcenkonflikte« durchführte.

Zahlreiche Mitglieder des ZeDF engagieren sich zudem als Lehrende im international ausgerichteten Masterstudiengang »Demokratisches Regieren und Zivilgesellschaft«, der das Verhältnis von Staat und Zivilgesellschaft im Kontext einer demokratischen Regierungspraxis thematisiert. Deren Beitrag zum gesellschaftlichen Frieden und zur politischen Problemlösungsfähigkeit auf lokaler, regionaler, nationaler und supranationaler Ebene ist Gegenstand einer vertieften politikwissenschaftlichen Analyse und Bewertung. Besondere Aufmerksamkeit erfahren dabei Praktiken und Strategien politischer Konfliktbearbeitung, die sich im Spannungsfeld zwischen kultureller Vielfalt und universellen Grundwerten sowie zwischen vielfältigen gesellschaftlichen Ansprüchen und ökonomischen Handlungsrestriktionen herausbilden. Am ZeDF angesiedelt ist schließlich auch das von der Deutschen Forschungsgemeinschaft (DFG) geförderte Forschungsprojekt »Securi- 
ty Governance durch Milizen« (2012-15) (Projektleitung: Prof. Dr. Ulrich Schneckener).

Als kleinere universitäre Initiative finanziert sich das ZeDF bisher fast ausschließlich aus den Mitteln der beteiligten Lehrstühle. Für die Zukunft beabsichtigt das Zentrum daher, neue interdisziplinäre Forschungsvorhaben und gemeinsame Projekte zu entwickeln sowie Initiativen in der akademischen Lehre - etwa der Nachwuchsförderung im Bereich der Friedens- und Konfliktforschung - neue Impulse zu verleihen.

\section{Kontakt}

Zentrum für Demokratie- und Friedensforschung (ZeDF)

Seminarstraße 33, Raum 04/214

49069 Osnabrück

Tel.: (0049) (0)541 969-4245

E-Mail: sekretariat.zedf@uni-osnabrueck.de

Homepage: www.zedf.uni-osnabrueck.de

\section{Der Autor}

Dr. phil. Bernhard Rinke ist wissenschaftlicher Mitarbeiter der Professur für Internationale Beziehungen und Friedens- und Konfliktforschung der Universität Osnabrück und Geschäftsführer des Zentrums für Demokratie- und Friedensforschung.

E-Mail: brinke@uni-osnabrueck.de 